\title{
ANÁLISIS
}

\section{ÉTICA DE LA PUBLICACIÓN CIENTÍFICA: INICIATIVAS Y RECOMENDACIONES}

\author{
Victoria Tur-Viñes, María-Carmen Fonseca-Mora y Begoña Gutiérrez-San- \\ Miguel
}

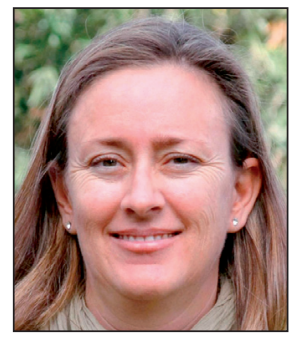

Victoria Tur-Viñes, profesora titular del Depto. de Comunicación y Psicología Social de la Univ. de Alicante (UA), es editora de Revista mediterránea de comunicación, presidenta de Plataforma Latina de Revistas de Comunicación y miembro del equipo investigador del Observatorio de Revistas Científicas de Ciencias Sociales. Sus líneas de investigación son comunicación científica, creatividad publicitaria y comunicación e infancia. Autora de varios libros y artículos en revistas indexadas. Pertenece a los comités científicos de revistas españolas y portuguesas del área de comunicación.

Universidad de Alicante Campus de S. Vicente del Raspeig, Edif. de Ciencias Sociales Ap. 99. 03080 Alicante, España victoria.tur@ua.es

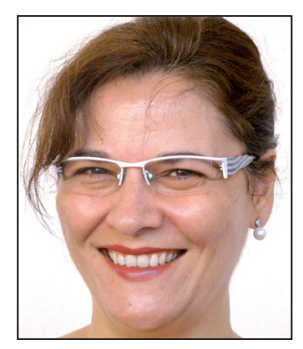

María-Carmen Fonseca-Mora es profesora en el Depto. de Filología Inglesa de la Univ. de Huelva (UHU), donde también es vicerrectora de Formación Permanente e Innovación. Investiga en lingüística aplicada y formación de profesores de idiomas. Sus trabajos han sido publicados en revistas académicas y volúmenes editados por Heidelberg Verlag, Netbiblio, Cambridge Scholars y Oxford Journals, entre otros. Es autora de varias publicaciones sobre revistas científicas. Ha impartido conferencias en Alemania, Portugal, Inglaterra, Suecia, Perú, Estados Unidos y España. Es editora adjunta de Comunicar y vicepresidenta de la Red Española de Revistas Científicas de Educación.

Universidad de Huelva Dr. Cantero Cuadrado, 6. 21071 Huelva, España fonseca@uhu.es

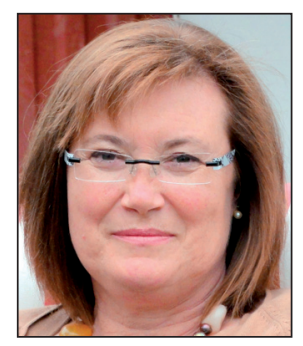

Begoña Gutiérrez-San-Miguel es profesora titular de la Universidad de Salamanca (USAL), en la licenciatura de Comunicación Audiovisual, impartiendo la asignatura de Narrativa Audiovisual. Es editora de la revista Fonseca journal of communication. Sus líneas de investigación giran en torno al lenguaje narrativo audiovisual, cinematografía, nacionalismo e identidad a través de lo audiovisual y contenidos narrativos televisivos. Es autora de varias publicaciones como Teoría de la narración audiovisual.

Universidad de Salamanca, Facultad de Ciencias Sociales Campus Miguel de Unamuno, 37007 Salamanca, España bgsm@usal.es

\section{Resumen}

La creciente importancia de las publicaciones científicas de calidad, su impacto en la sociedad en general y en los procesos de acreditación de los autores, así como la necesidad de visibilidad internacional, pueden provocar un incremento de los conflictos de intereses entre editores, autores y revisores de publicaciones científicas. Se revisan las recomendaciones éticas existentes. Se analizan las principales iniciativas internacionales, los organismos promotores y las recomendaciones específicas que se realizan para cada agente del proceso. El grado de sensibilización frente a la ética de las publicaciones en cada ciencia y país depende de la tradición, el asociacionismo, la reputación y el grado de internacionalización de las revistas que configuran cada campo científico.

\section{Palabras clave}

Ética, Publicación científica, Código de conducta, Buenas prácticas, Editores, Autores, Revisores.

Title: Ethics of scientific publishing: initiatives and recommendations 


\begin{abstract}
The growing importance of scientific publications of quality, their impact on society in general and on authors' processes of scientific recognition, as well as journals needing to have international visibility, may increase the number of conflicts of interests among editors, authors and reviewers of scientific publications. The aim of this article is to review recommendations of existing ethics codes. Major international initiatives, their sponsoring entities and specific recommendations made to the different agents of the process are analyzed. The review reveals that the degree of awareness about the ethics of the publications in each discipline and each country depends on tradition, associations, the established reputation and the degree of internationalization of the journals that make up each scientific field.
\end{abstract}

\title{
Keywords
}

Ethics, Scientific publication, Code of conduct, Good practices, Editors, Authors, Reviewers.

Tur-Viñes, Victoria; Fonseca-Mora, María-Carmen; Gutiérrez-San-Miguel, Begoña. "Ética de la publicación científica: iniciativas y recomendaciones". El profesional de la información, 2012, septiembre-octubre, v. 21, n. 5, pp. $491-497$.

\section{Introducción}

La publicación científica es la práctica más extendida para compartir los resultados de la investigación. Es considerada "un instrumento de evaluación: determina el ascenso en la escala profesional y social de los científicos e influye decisivamente en la asignación de recursos económicos para la investigación" (Delgado-López-Cózar, 2001, p. 47). Sin embargo, una publicación en una revista científica puede no asegurar que lo difundido proceda de investigaciones solventes, autores responsables, evaluadores competentes y editores imparciales. El caso Hwang en la revista Science descrito por Delgado-López-Cózar, Torres-Salinas y RoldánLópez (2007) donde los datos fueron inventados y nunca se llegó a clonar un embrión, o el reciente caso Doñana ${ }^{1}$ en el que un investigador alteró e inventó datos en diferentes estudios sobre aves durante años, reclaman mecanismos basados en la transparencia, veracidad y credibilidad de la publicación.

La existencia de un código ético manifiesta la concienciación, asegura la prevención del fraude científico y orienta sobre el procedimiento en casos de mala praxis.

La existencia de un código ético manifiesta la concienciación, asegura la prevención del fraude científico y orienta sobre el procedimiento en casos de mala praxis

\section{Orígenes de la ética de las publicaciones en la literatura científica}

Los códigos éticos de publicaciones científicas surgen en las décadas de los años 70 y 80 en los EUA por la denuncia pública de investigaciones fraudulentas ${ }^{2}$. La Agencia Federal y el Congreso ejercieron un serio control sobre las investigaciones científicas (LaFollette, 2000), que fue percibido por la comunidad científica como una intolerable intromisión. Su protesta promovió la creación de la Office of Research Integrity $(O R I)$ en la década de los 90 . Posteriormente asociacio- nes americanas de editores de revistas médicas, sociedades científicas de química, física y matemáticas comenzaron a publicar sus códigos éticos. En Europa nacen los distintos consejos y comités éticos entre finales de los 90 y la primera década del s. XXI. Sin embargo, Bosch (2011) denuncia que ni la Comisión Europea en su programa de financiación de la investigación Horizon 2020, ni el Código Europeo de Conducta para la Integridad de la Investigación han establecido aún recomendaciones específicas para evitar y/o penalizar el fraude.

\section{Organismos promotores}

La mayoría de iniciativas observan la ética de la investigación (ORI, Equator Network, Csic). La publicación científica como actividad investigadora aparece implícita. Otros organismos se concentran en la actividad editora: Committee on Publication Ethics (Cope), Council of Science Editors (CSE), World Association of Medical Editors (Wame), International Committee of Medical Journal Editors (Icmje). También el proyecto Necobelac.

El origen es diverso. Encontramos sociedades científicas como American Psychological Association, American Physical Society, American Chemical Society, American Mathematical Society. Editoriales como Elsevier y Blackwell (Graf et al., 2007) estipulan requisitos uniformes para el envío de textos a sus revistas. La iniciativa pública también está presente (OR/3-EUA; Csic-España) actuando en conflictos. También hacen aportaciones redes como Necobelac, de colaboración entre Europa y América Latina y Caribe, destinada a difundir conocimientos sobre la escritura científica y el acceso a la publicación en ciencias de la salud, o Equator (Enhancing the Quality and Transparency of Health Research), un centro de recursos que contribuye a la comunicación de las investigaciones en salud. Analizamos ahora las iniciativas que realizan propuestas originales y no remiten a otros organismos.

\section{Committee on Publication Ethics (Cope)}

Promovido por ciertos organismos biosanitarios en 1999, hoy cuenta entre sus miembros con revistas de todos los campos científicos, entre ellas quince españolas. Cope ofrece diferentes grados de compromiso editorial: 
- Best practice guidelines for journal editors ${ }^{4}$, reemplazadas en 2006 por una serie de diagramas de flujo orientativos de la toma de decisiones para solucionar incidencias relacionadas con la publicación, traducidos a varios idiomas.

- Code of conduct ${ }^{5}$, elaborado en 2004 para editores, establecía normas para la buena redacción, apelaba a la responsabilidad de los editores, instaba a investigar pormenorizadamente los casos de mala praxis ofreciendo su mediación. Se actualizó en 2007 y en $2011^{6}$. Combina el código con una serie de recomendaciones de buenas prácticas que denomina "aspiracionales", reconociendo la dificultad de su cumplimiento íntegro. Se concentra en la función de los editores e incluye directrices para el resto de agentes pero sólo en lo que concierne a su relación con editores.

\section{Council of Science Editors (CSE)}

Council of Biology Editors, creado en 1957 por la acción conjunta de la National Science Foundation y el American Institute of Biological Sciences, cambió su denominación a Council of Science Editors (CSE) en 2000 para evidenciar su apertura a otras ciencias. El CSE's White paper on promoting integrity in scientific journal publications ${ }^{7}$ es la propuesta más reciente (marzo 2012). Introduce indicaciones sobre manipulación de la citación, planificación de la publicación por los patrocinadores y su conducta ética, la mejora de informes y documentos sobre textos sospechosos. Actualiza la información, identifica directrices para autores, editores, revisores, propietarios y grupos editoriales.

\section{International Committee of Medical Journal Editors (Icmje)}

Conocido originariamente como Grupo Vancouver, elaboró en 1979 los Uniform requirements for manuscripts submitted to biomedical journals ${ }^{8}$, actualizados anualmente, que incluyen los formatos de citación bibliográfica de la National Library of Medicine de EUA. Estipula principios éticos para la autoría y reconocimiento de colaboradores en publicaciones biomédicas, la responsabilidad de editores y la libertad editorial, la revisión por pares, los conflictos de interés, la privacidad y la confidencialidad.

\section{Comité de Ética del Consejo Superior de Investigacio- nes Científicas (Csic)}

En 2011 publicó el Código de buenas prácticas ${ }^{9}$, con recomendaciones para las publicaciones científicas: su difusión oral y escrita, publicación de resultados, autoría, reconocimiento de autores previos y revisión de originales. De carácter consultivo y permanente, el Comité se encarga de reflexionar, emitir informes y formular recomendaciones sobre los principios éticos y deontológicos relativos a la actividad investigadora.

\section{World Association of Medical Editors (Wame)}

Impulsa desde 1995 las Publication ethics policies for medical journals ${ }^{10}$. Suscribe las directrices Icmje añadiendo indicaciones específicas para el revisor, quien debe localizar y analizar las fortalezas y debilidades de la metodología, realizar comentarios constructivos y precisos que permitan mejorar el texto, denunciar las sospechas y preservar el anonimato, entre otras.

Otras iniciativas remiten a alguna de las expuestas:

- European Association of Science Editors (Ease) referencia en temas éticos a Cope;

- Office of Research Integrity (ORI) deriva de Icmje aunque para determinar el concepto de autoría se basa en CSE;

- World Association of Medical Editors (Wame) enfatiza en los revisores, comparte recomendaciones con Icmje dando unas indicaciones específicas para el revisor;

- Necobelac utiliza como material de apoyo los documentos de Cope y Wame;

- Strengthening the Reporting of Observational studies in Epidemiology (Strobe) recomienda las normas de Icmje;

- Equator Network, ya citada, surgió del grupo de trabajo Consolidated Standards of Reporting Trials en 2006. Recopila las instrucciones para evaluadores que ofrecen las revistas del ámbito biomédico y relaciona todas las iniciativas existentes a modo de información agrupada para editores;

- Consort promueve Assert ${ }^{11}$ como directrices para los ensayos clínicos y Prisma ${ }^{12}$ (antes Quorum), que orienta sobre los textos de revisión y meta-análisis.

Las propuestas analizadas concretan recomendaciones éticas para autores, editores, propietarios y grupos editoriales, patrocinadores, evaluadores y lectores que adoptan la forma de códigos o directrices de buenas prácticas. Proporcionan también orientación sobre la forma de proceder en casos de conflicto o mala praxis. El material de apoyo diseñado en el proyecto Necobelac ${ }^{13}$ sintetiza los casos más comunes: redundancia o publicación duplicada, plagio, falsificación de datos, conflicto de intereses y problemas de reconocimiento de autoría. 


\begin{tabular}{|l|l|l|l|l|l|}
\hline Organismo & $\begin{array}{l}\text { Committee on } \\
\text { Publication Ethics (Cope) }\end{array}$ & $\begin{array}{l}\text { Council of Science Editors } \\
\text { (CSE) }\end{array}$ & $\begin{array}{l}\text { International Committee } \\
\text { of Medical Journal Editors } \\
\text { (Icmje) }\end{array}$ & $\begin{array}{l}\text { Consejo Superior } \\
\text { de Investigaciones } \\
\text { Científicas } \\
\text { (Csic) }\end{array}$ & $\begin{array}{l}\text { World } \\
\text { Association of } \\
\text { Medical Editors } \\
\text { (Wame) }\end{array}$ \\
\hline $\begin{array}{l}\text { Documento } \\
\text { analizado }\end{array}$ & $\begin{array}{l}\text { Code of conduct. Best } \\
\text { practice guidelines for } \\
\text { journal editors }\end{array}$ & $\begin{array}{l}\text { CSE's White paper on } \\
\text { promoting integrity in } \\
\text { scientific journal publications }\end{array}$ & $\begin{array}{l}\text { Uniform requirements for } \\
\text { manuscripts submitted to } \\
\text { biomedical journals }\end{array}$ & $\begin{array}{l}\text { Código de buenas } \\
\text { prácticas }\end{array}$ & $\begin{array}{l}\text { Publication } \\
\text { ethics policies for } \\
\text { medical journals }\end{array}$ \\
\hline País impulsor & Reino Unido & EUA & Canadá & España & EUA \\
\hline $\begin{array}{l}\text { Año del } \\
\text { documento }\end{array}$ & 2011 & 2012 & 2010 & 2011 & 1995 \\
\hline $\begin{array}{l}\text { Ámbito } \\
\text { científico } \\
\text { impulsor }\end{array}$ & $\begin{array}{l}\text { CC. de la salud. } \\
\text { Actualmente } \\
\text { multidisciplinar. }\end{array}$ & $\begin{array}{l}\text { Biomedicina. Actualmente } \\
\text { multidisciplinar }\end{array}$ & Biomedicina & Bioética & Medicina \\
\hline
\end{tabular}

Tabla 1: Comparativa de las propuestas originales analizadas

\section{Recomendaciones éticas}

Varias investigaciones (Wager et al., 2009; Angelski et al., 2012) describen la percepción de editores de revistas científicas en lengua inglesa sobre posibles malas praxis. En ambos estudios respondió cerca del $45 \%$ de los encuestados, mayoritariamente de ciencias de la salud, afirmando que rechazaban manuscritos sospechosos pero no daban instrucciones específicas a sus revisores. La preocupación es evitar la duplicación de artículos, las publicaciones redundantes y determinar los criterios de autoría. La mayoría de los editores desconocía las directrices sobre buenas prácticas o los códigos de los organismos citados, aunque sí las instrucciones de otras revistas.

Por otro lado, estudios como los de Rowan-Legg et al. (2009), Callaham y Tercier (2007) o Tavares-de-Matos-Cardoso (2011) muestran que las instrucciones éticas tanto para autores como para revisores son aún insuficientes y las que existen, requieren mayor difusión y estandarización. Resnik, Patrone y Peddada (2010) señalan que el impacto de una revista se relaciona con la existencia de una política editorial explícita sobre mala praxis.

A continuación exponemos las recomendaciones específicas emitidas por los organismos revisados, dirigidas a:

\section{Editores y grupos editoriales}

\section{Responsabilidad}

Los editores son responsables de lo que se publica en la revista y de los procesos implícitos. Si existe un propietario o

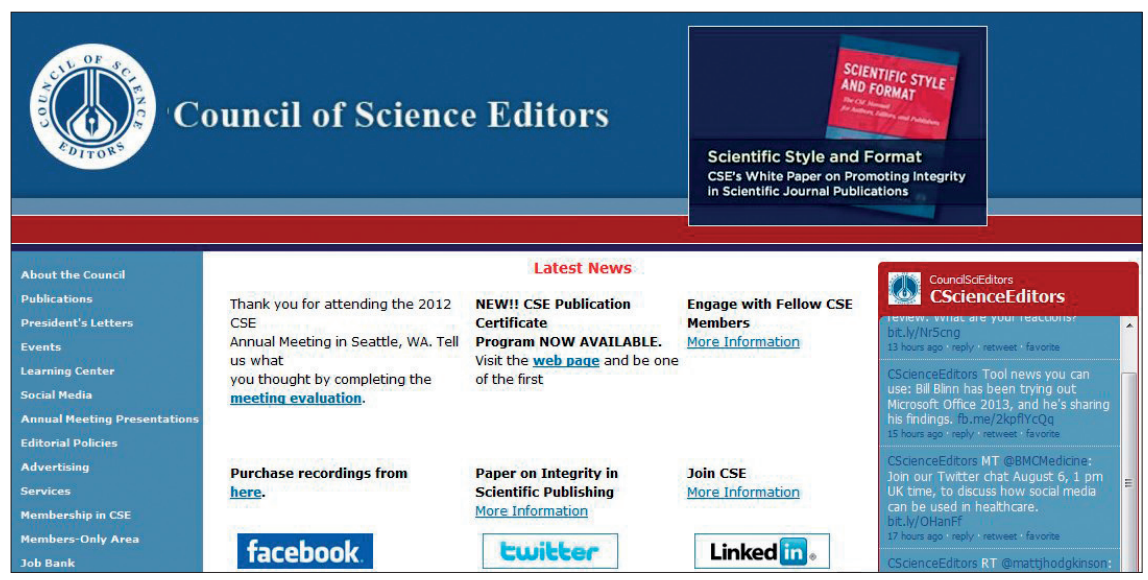

Council of Science Editors (CSE) grupo editorial, tanto Cope como CSE recomiendan la existencia de un contrato que refleje las obligaciones y derechos de editores. CSE sugiere concretar las expectativas sobre su trabajo, los objetivos a alcanzar y los sistemas previstos para la auditoría externa de su actividad. En casos de mala praxis los editores deben ser proactivos, denunciar y resolver el conflicto interactuando con los afectados.

\section{Libertad editorial}

Los editores deben contar con la máxima libertad para gestionar su trabajo. Cope considera un deber de los editores el preservar la libertad de expresión de los autores.

\section{Confidencialidad en todo el proceso}

Todos coinciden en la importancia de preservar el anonimato en el proceso de revisión por pares.

Actuación en casos de conflicto de intereses en que existen circunstancias (personales, académicas, políticas, financieras o religiosas) que pueden sesgar la decisión de los editores.

CSE recomienda proceder según los diagramas de flujo de Cope. El conflicto de intereses es perjudicial para la reputación de una revista. Es deseable realizar las rectificaciones, comunicaciones o aclaraciones que procedan y vincularlas adecuadamente al artículo relacionado, como medida disuasoria y/o sanción.

\section{Manipulación de citas}

CSE contempla los casos de coacción (si los editores solicitan al autor la inclusión de citas de artículos de su propia revista), editoriales que referencian artículos de la propia revista, la auto-citación y la cita de intercambio (compromiso de citación entre un grupo de autores). Se recomienda la condena de estas prácticas en las normas de la revista.

\section{Coordinación}

Los editores estipulan el funcionamiento del comité editorial y velan por el cumplimiento de sus funciones (revisión de manuscritos, asignación de evaluadores y asesoramiento en la delimitación de la política editorial). 


\section{Gestión}

El registro detallado de todas las interacciones es necesario para cualquier auditoría externa y para el propio control interno de los procesos.

\section{Disputas de autoría}

Los editores deben publicar orientaciones al respecto, suspendiendo el proceso de revisión o la publicación del texto hasta que se resuelva.

\section{Vías de reclamación}

Se recomienda a los editores publicarlas y motivar adecuadamente las decisiones.

\section{Preservación de los derechos}

Se recomienda claridad en la declaración de los derechos de explotación (copyright). Con el alza del open access y el auto-archivo se hace necesario dar a conocer el detalle de los tipos de licencias (Melero, 2010).

Estímulo de la investigación ética que implique a personas o animales

La investigación publicada debe estar sujeta a directrices internacionalmente aceptadas (Declaración de Helsinki para la investigación clínica; American Educational Research Association (Aera) y las directrices Ethical Guidelines for Educational Research (Bera) para la investigación educativa). Cope recomienda el debate científico a partir de los textos publicados y reconoce el interés que reviste la publicación de resultados negativos o investigaciones fallidas para el avance científico.

La responsabilidad moral y ética de los autores implica consistencia y fiabilidad de la investigación, honestidad, originalidad, responsabilidad y transparencia

\section{Patrocinadores, anunciantes y lectores}

Se recogen en Cope dentro de las indicaciones en la relación del editor con los lectores. Los lectores deben estar informados sobre quién financia la investigación o el estudio y qué papel ha ostentado. Se encuentran indicaciones específicas exhaustivas sobre la publicidad y los patrocinadores. No son admisibles los textos que describan las propiedades o efectos de un producto que esté distribuido o fabricado por un patrocinador de la revista.

\section{Autores}

EI CSE, Cope e Icmje delimitan el concepto de autor estableciendo los siguientes criterios:

a) El grado de participación en el estudio dependerá de la presencia simultánea de estas condiciones: la intervención en todas las fases (estructura, redacción y revisión), la coordinación con el resto de autores, la contribución a la obtención de fondos, la recopilación y el análisis de datos.

b) El orden de firma se decidirá de forma consensuada y previa. Kassirer (1995) define al autor como el que asume la responsabilidad pública del contenido del artículo. Los investigadores con contribuciones menores solamente aparecerían en los agradecimientos.

c) La responsabilidad moral y ética de los autores implica:

- Consistencia y fiabilidad en la investigación: ha de ser responsable y legal, cuidadosamente trabajada, utilizar métodos de análisis correctos, garantizar los resultados y presentarla adecuadamente.

- Honestidad: sin mentiras ni falsificaciones o manipulación de datos.

- Original: no publicada en otro país o idioma, no presentada simultáneamente a más de una publicación sin que los editores hayan acordado la co-publicación señalándolo en el texto, sujeta a las leyes de propiedad intelectual citando fuentes primarias identificables.

- Transparente con las fuentes de financiación de la investigación, incluyendo el apoyo financiero directo e indirecto, el suministro de equipos o materiales u otro tipo de apoyo.

- Responsabilidad: los autores deberán ajustar el texto a las normas de publicación establecidas. Deben colaborar haciendo rápidas y ágiles las correcciones. Deben responder a la aceptación o rechazo y a las sugerencias de los revisores.

\section{Evaluadores}

Tanto CSE como Wame y el resto de organismos que deriva de ellos destacan el carácter voluntario y la necesaria 
especialización de los revisores. La revisión científica más extendida es por pares y anónima (simple o doble ciego), determina la reputación de una revista e influye en su factor de impacto. En casos de controversia o desacuerdo deciden los editores.

El revisor tiene responsabilidades con:

\section{-Los autores}

Proporciona información objetiva por escrito sobre la valoración científica del trabajo, la forma de escritura, la precisión, la originalidad e interés, evitando comentarios discutibles o críticas innecesarias, y sobre todo no exigiendo más de lo que él mismo estaría dispuesto a hacer.

\section{-Los editores}

Notifica al editor si puede o no hacer la revisión y proporciona los nombres de revisores alternativos. Alerta sobre posibles conflictos personales o de intereses y rechaza la revisión en su caso. Debe realizar una crítica justa y constructiva, incluyendo material complementario, indicando la manera de mejorar el texto y recomendando su aceptación o rechazo. Debe denunciar la ruptura de las normas éticas (duplicación, no inédito...), preservar la confidencialidad y el anonimato.

\section{-Los lectores}

Garantizando que se citan todos los trabajos pertinentes de otros científicos.

Las responsabilidades éticas están basadas en la confidencialidad, la crítica constructiva, la competencia, la imparcialidad e integridad, la divulgación de conflicto de intereses y el respeto a los plazos para completar la revisión. Wame e Icmje precisan reglas éticas para el revisor, en la línea de las recomendaciones enumeradas. ORI y CSE inciden en el anonimato de la revisión y debaten sobre la fórmula óptima: pares parcialmente enmascarados o bien el sistema abierto con revelación de identidades.

\section{Conclusiones}

Las recomendaciones éticas para la publicación científica se derivan de una preocupación previa por la ética de la investigación. La publicación es una consecuencia de la investigación pero no siempre tiene un tratamiento específico suficiente. Las iniciativas éticas que adoptan un enfoque multidisciplinar y compilan recomendaciones para todos los agentes del proceso (Cope y CSE) resultan especialmente orientadoras. Falta precisión en algunos de los enunciados de las normas (por ejemplo, una recomendación que afecta a editor y revisor sólo aparece en las recomendaciones al editor) y una adaptación realista al agente involucrado.

Coexisten iniciativas similares, la mayoría foráneas y en lengua inglesa, y adaptadas a un único campo científico, mayoritariamente biomédico. La transparencia y la convergencia en temas éticos se imponen como necesidades ineludibles que contribuyan a clarificar y mejorar el ejercicio honesto de la publicación.

El grado de sensibilización frente a la ética de las publicaciones en cada ciencia y en cada país parece depender de la tradición, el asociacionismo, la consolidación y el grado de internacionalización de las revistas que configuran cada campo científico.

\section{Se percibe desinformación, insuficiente concienciación y un tratamiento no es- pecífico del tema ético entre editores, revisores y autores}

Se percibe insuficiente concienciación, cierta desinformación y un tratamiento no específico del tema ético entre editores, revisores y autores, especialmente en el área de las revistas de ciencias sociales. Las recomendaciones éticas no tienen entidad propia en la política editorial de las revistas y aparecen diseminadas en las distintas normas. Para comprobar esta hipótesis, una vez identificadas las principales recomendaciones, nos proponemos realizar en el futuro un estudio que permita conocer cómo son contempladas en las revistas españolas de cada campo científico y profundizar en las revistas de ciencias sociales realizando estudios comparativos.

\section{Notas}

1. Caso Doñana: http://sociedad.elpais.com/sociedad/2012/06/11/actualidad/1339441618_930007.html

2. Caso Breuning: Valentine, Paul W. "Drug therapy researcher is indicted". Washington Post, 1988, 111, pp. A1, A14.

3. ORI está vinculada a la Office of Public Health and Science (OPHS) dependiente de la Office of the Secretary of Health and Human Services (OS) en el Department of Health and Human Services (HHS) de EUA.

4. http://publicationethics.org/static/1999/1999pdf13.pdf

5. http://publicationethics.org/files/2008 Code of Conduct. $p d f$

6. http://publicationethics.org/files/Code of conduct for journal editors_0.pdf

7. http://www.councilscienceeditors.org/files/public/entire_whitepaper.pdf

\section{Agradecimiento}

A Remedios Melero (CSIC) por su orientación e indicaciones para la localización de algunos documentos.

\section{Financiación}

Este estudio ha sido financiado por el Ministerio de Ciencia e Innovación de España, dentro del Plan Nacional de I+D+i (EDU2011-13034-E), acción complementaria: Observatorio de revistas científicas de ciencias sociales.

\section{Direcciones de interés}

Committee on Publication Ethics (Cope)

http://publicationethics.org 
Comité de ética del Csic

http://www.csic.es/web/guest/etica-en-la-investigacion;jse ssionid=FC6C6C5622CE1605573A349A25194596

Council of Science Editors (CSE)

http://www.councilscienceeditors.org/i4a/pages/index. cfm?pageid $=3636$

World Association of Medical Editors (Wame)

http://www.wame.org

The Equator Network-Consort

http://www.equator-network.org

European Association of Science Editors (Ease)

http://www.ease.org.uk

Office of Research Integrity (ORI)

http://ori.hhs.gov

Network of Collaboration Between Europe \& Latin American-Caribbean countries (Necobelac)

http://62.204.194.27/necobelac/generalesp.jsp?id=id7360

American Psychological Association

http://www.apa.org

American Physical Society

http://www.aps.org

American Chemical Society

http://portal.acs.org/portal/acs/corg/content

American Mathematical Society

http://www.ams.org/home/page

Strengthening the Reporting of Observational studies in Epidemiology (Strobe)

http://www.strobe-statement.org

Autores, editores, revisores: las reglas del juego.

http://www.elsevier.es/es/revistas/gaceta-sanitaria-138/

autores-revisores-editores-las-reglas-juego-13018922-notaeditORIal-2001

\section{Bibliografía}

Angelski, Carla L.; Fernández, Conrad V.; Weijer, Charles; Gao, Jun. "The publication of ethically uncertain research: attitudes and practices of journal editors". BMC medical ethics, 2012, v. 13, n. 4.

http://www.biomedcentral.com/content/pdf/1472-693913-4.pdf http://dx.doi.org/10.1186/1472-6939-13-4

Bosch, Xavier. "Scientific fraud: Europe must address research misconduct". Nature, 2011, v. 480, n. 7376, p. 181. http://dx.doi.org/10.1038/480181c

Callaham, Michael L.; Tercier, John. "The relationship of previous training and experience of journal peer reviewers to subsequent review quality". PLoS med, 2007, v. 4, n. 1, e40. http://www.plosmedicine.org/article/info:doi/10.1371/ journal.pmed.0040040

http://dx.doi.org/10.1371/journal.pmed.0040040

Delgado-López-Cózar, Emilio. “Las revistas españolas de ciencias de la documentación: productos manifiestamente mejorables". El profesional de la información, 2001, diciembre, v. 10, n. 12, pp. 46-56.
http://ec3.ugr.es/publicaciones/Las_revistas_espannolas de_Biblioteconomia_y_Documentacion.pdf

Delgado-López-Cózar, Emilio; Torres-Salinas, Daniel; Roldán-López, Álvaro. "El fraude en la ciencia: reflexiones a partir del caso Hwang". El profesional de la información, 2007, marzo-abril, v. 16, n. 2, pp. 143-150.

http://ec3.ugr.es/publicaciones/El_fraude_en_la_ciencia. $p d f$

http://dx.doi.org/10.3145/epi.2007.mar.07

Graf, Chris; Wager, Elizabeth; Bowman, Alyson; Fiack, Suzan; Scott-Lichter, Diane; Robinson, Andrew. "Best practice guidelines on publication ethics: a publisher's perspective". International journal of clinical practice, 2007, n. 61, pp. 126.

http://www.ncbi.nlm.nih.gov/pmc/articles/PMC1804120 http://dx.doi.org/10.1111/j.1742-1241.2006.01230.x

Kassirer, Jerome. "Authorship criteria". Science, 1995, n. 268, pp. 785-786.

LaFollette, Marcel C. "The evolution of the 'scientific misconduct': an historical overview". Exp biol med, 2000, v. 224, n. 4, pp. 211-215.

http://ebm.rsmjournals.com/content/224/4/211.full. pdf+html

Melero, Remedios. Guía práctica sobre los derechos patrimoniales o de explotación (copyright) y su relación con el auto-archivo en repositorios de acceso abierto, 2010.

http://www.accesoabierto.net/node/62

Resnik, David B.; Patrone, Daniel; Peddada, Shyamal. "Research misconduct policies of social science journals and impact factor". Accountability in research: policies and quality assurance, 2010, v. 17, n. 2, pp. 79-84.

http://www.ncbi.nlm.nih.gov/pmc/articles/PMC3065865/ ?tool=pubmed

http://dx.doi.org/10.1080/08989621003641181

Rowan-Legg, Anne; Weijer, Charles; Gao, Jun; Fernández, Conrad. "A comparison of journal instructions regarding institutional review board approval and conflict-of-interest disclosure between 1995 and 2005". Journal of medical ethics, 2009, n. 35, pp. 74-78.

http://dx.doi.org/10.1136/jme.2008.024299

Tavares-de-Matos-Cardoso, Manuela. "El peer review de las revistas científicas en humanidades y ciencias sociales: políticas y prácticas editoriales declaradas". Revista española de documentación científica, 2011, v. 34, n. 2, pp. 141164.

http://redc.revistas.csic.es/index.php/redc/article/ viewArticle/690

http://dx.doi.org/10.3989/redc.2011.2.796

Wager, Elizabeth; Fiack, Suzan; Graf, Chris; Robinson, A.; Rowlands, I. "Science journal editors' views on publication ethics: results of an international survey". Journal of medical ethics, 2009, v. 35, n. 6, pp. 348-353.

http://jme.bmj.com/content/35/6/348.full.pdf+html

http://dx.doi.org/10.1136/jme.2008.028324 